LA-UR-01-3187

Approved for public release; distribution is unlimited.

\section{Simulated, Theoretical and Experimental Shock Trajectories in Cylindrical Geometry}

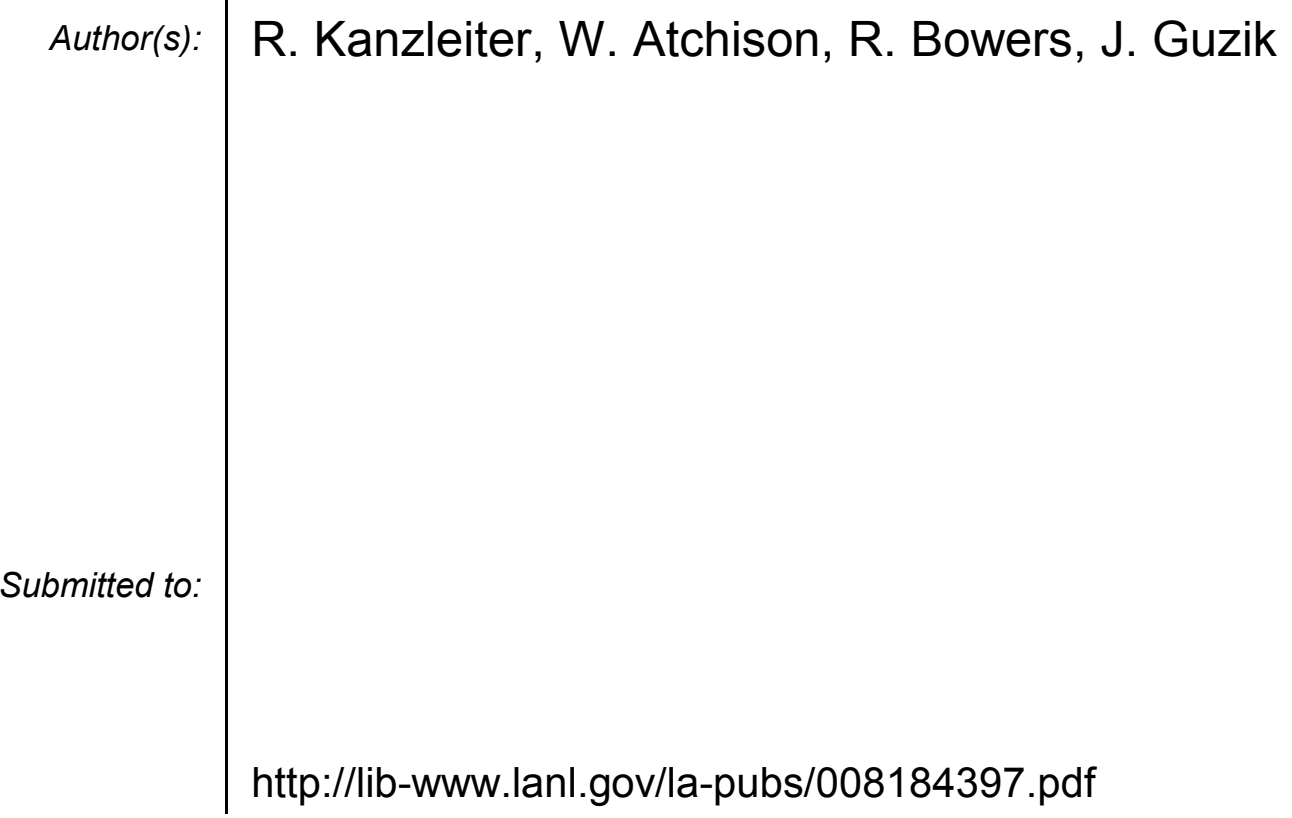




\title{
Simulated, Theoretical and Experimental Shock Trajectories in Cylindrical Geometry
}

\author{
R. Kanzleiter, W. Atchison, R. Bowers, J. Guzik \\ Los Alamos National Laboratory, MS B-220 \\ Los Alamos, NM 87545
}

\begin{abstract}
The current work compares computations and similarity relations for convergent shocks with experimental data from cylindrical implosions on the Shiva Star capacitor bank at AFRL (Air Force Research Laboratory). The experiment consisted of a $1 \mathrm{~mm}$ thick cylindrical aluminum liner that is magnetically imploded onto a central target. The central target contains an inner Lucite cylinder surrounded by an outer Sn layer. The target was designed using the Eulerian hydrodynamics code RAGE in 2- and 3D. One-dimensional models of the liner driver utilizing the RAVEN MHD code set the initial liner/target interaction parameters for the RAGE simulations. Shock breakout from the $\mathrm{Sn} /$ Lucite interface and subsequent propagation within the Lucite are measured to provide experimental data for code validation. Radial convergence is examined through comparisons with similarity solutions. Comparison of experimentally measured timing data and simulated shock trajectories will be discussed. Further efforts compare equation-of-state effects by utilizing an analytic Grueneisen EOS instead of the original SESAME tables.
\end{abstract}

\section{INTRODUCTION}

As hydrodynamic simulations become more sophisticated, it is necessary to validate that codes in general use can predict relatively simple phenomena let alone complex problems. Towards this end, converging shock waves in cylindrically symmetric geometries are examined from a computational, experimental and theoretical point of view. We find that even in the most idealized cases no exact analytical solution exists for the behavior of converging shocks. This necessitates that code validation efforts must eventually focus on comparisons of simulations with high quality experimental data. Efforts are currently underway to design pulsed power experiments and obtain such data characterizing hydrodynamic phenomena in convergent geometries. As a preliminary step, a series of Near Term Liner eXperiments (NTLX) was designed and fielded on the Shiva Star Pulsed Power Machine at AFRL. We find generally good agreement between the codes employed and the theoretical/measured data except in the near axis region where convergence dominates.

\section{COMPUTATIONAL TOOLS}

Experiment design and hydro calculations were performed using the adaptive mesh refinement (AMR) Eulerian hydrodynamics code RAGE ${ }^{1}$. This is a 1-, 2- or 3D, multi-material Eulerian hydro code utilizing adaptive mesh refining (AMR) and either analytic or tabular equations-of-state data. Code features and assumptions employed within RAGE include: 2D cartesian and cylindrical coordinate systems, 3D cartesian coordinate system, continuous adaptive mesh refinement, arbitrary number of materials per cell, pressure and temperature equilibrium for all materials in a cell, higher order piecewise linear Godunov numerical method, exact conservation of mass, momentum and total energy and dynamic program memory 
allocation. RAGE does lack the electromagnetic effects describing pulsed power magnetic drives such as Shiva Star, so the one-dimensional Lagrangian code RAVEN ${ }^{2,3}$ was used to model MHD effects and to set the initial liner/target interaction parameters.

RAVEN is a 1D Langrangian MHD code utilizing artificial viscous stresses to represent shocks and $\mathrm{SESAME}^{4}$ equation-of-state data to model the liner driver and subsequent shock evolution. The pulsed power drive for the NTLX experiments is represented through a self-consistent circuit model employing a lumped parameter RLC circuit. This circuit model allows for selfconsistent calculations of coupling between the pulsed power system and the imploding liner driver. RAVEN includes a Steinberg-Guinan ${ }^{5}$ strength model along with a tabular melt model for the $\mathrm{Al}$ liner driver. Resistivity data from SESAME completes the circuit model and determines the liner state at impact.

\section{EXPERIMENTAL ARRANGEMENT}

The NTLX series employed a previously characterized cylindrical Al liner driver that is magnetically imploded onto a central target. Following current initiation, the self-induced radial Lorentz force accelerates the $1 \mathrm{~mm}$ thick aluminum liner to a radial velocity of $6.25 \mathrm{~mm} / \mu \mathrm{s}$ at a target radius of $2 \mathrm{~cm}$. The convergent shock generated at liner/target impact drives subsequent hydrodynamic experiments to characterize shock behavior in various target geometries. The target conFiguration for the NTLX series contained two materials. An outer Sn layer was used to isolate convergence effects from the magnetic drive. For shock pressures greater than approximately $300 \mathrm{kbar}$ the Sn melts, thereby removing complications associated with material strength from comparisons. The inner core of the target was a Lucite cylinder to facilitate visualization of both the transmitted shock and the $\mathrm{Sn} /$ Lucite material interface. Figure 1 shows the symmetric $\mathrm{Sn} /$ Lucite target used during this experiment.

Visualization of the shock/interface behavior and timing of the liner/target impact was accomplished by axial radiography and a series of B-dot probes respectively. The B-dot probes measured highly

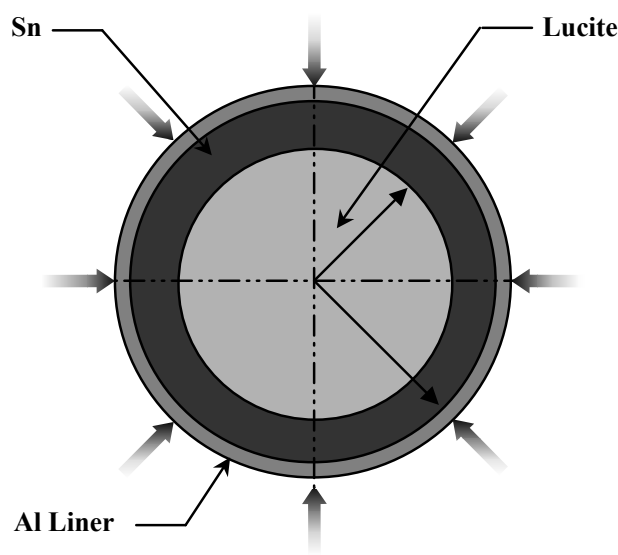

FIGURE 1. The target consisted of an inner Lucite core surrounded by an outer Sn layer. Later conFigurations explored off-center shock convergence by shifting the center of the Lucite target from the line driver center.

symmetric liner impacts with symmetries, $\Delta \mathrm{r} / \mathrm{r}$, between $97.5 \rightarrow 99 \%$ at a target radius of $2 \mathrm{~cm}$. Radiographically, image resolutions on the order of $0.09 \mathrm{~mm}$ were routinely obtained for analysis of the shock and interface locations.

\section{THEORETICAL BEHAVIOR}

An exact analytical solution does not exist for shocks propagating in convergent geometries. Instead, a large volume of work exists describing various similarity based semi-analytic solutions in cylindrical or spherical geometry ${ }^{6,7,8}$. The solutions examined to date have invariably simplified the problem to a calorically perfect, ideal gas. For sufficiently strong shocks, the convergent motion becomes self-similar as

$$
\boldsymbol{R}(\boldsymbol{t})=\boldsymbol{C} \cdot \boldsymbol{t}^{\alpha}
$$

where $\boldsymbol{C}$ and $\alpha$ are constants determined by numerical integration of the equations of motion. One general problem with these methods is that the assumptions inherent in eq. 1 break down for shocks that are too strong or in the near axis region where strengthening occurs due to area convergence effects. 


\section{RESULTS}

A first comparison between semi-analytic and simulation results considers the simplified problem of a mach 2 shock converging in a $2 \mathrm{~cm}$ radius column of argon at STP (standard temperature and pressure). RAGE simulations utilizing both SESAME and ideal gas EOS information are compared to self-similar results for a $\gamma=5 / 3$ gas in Fig. 2. Excellent agreement is obtained for the shock trajectory with a slight divergence between the simulation and similarity results near the convergence axis. This behavior is expected in that the assumptions inherent in the self-similar results are noted to break down near axis.

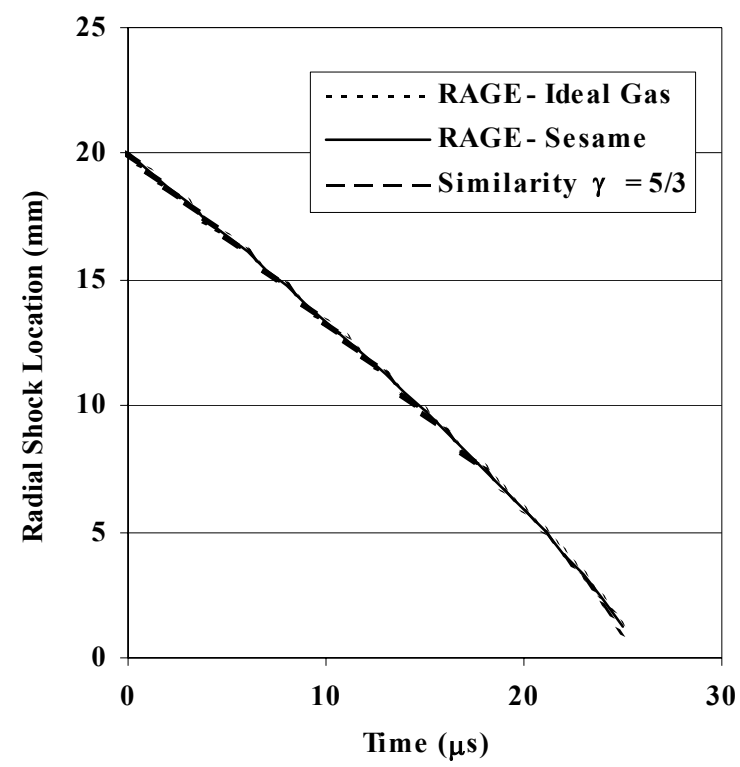

FIGURE 2. Self-similar and simulated results for a $M=2$ shock show excellent agreement in the shock trajectories.

As the strength of the incident shock increases, the ideal gas assumption underlying the self-similar solution and the RAGE - Ideal Gas result breaks down. Figure 3 shows a mach 10 shock converging in the same $2 \mathrm{~cm}$ STP argon cylinder as in Fig. 2. Compared to the $\mathrm{M}=2$ case, Fig. 3 exhibits a larger level of discrepancy among all calculations. Examination of even higher strength shocks shows a trend of increasing divergence between the simulation and similarity-based solutions.

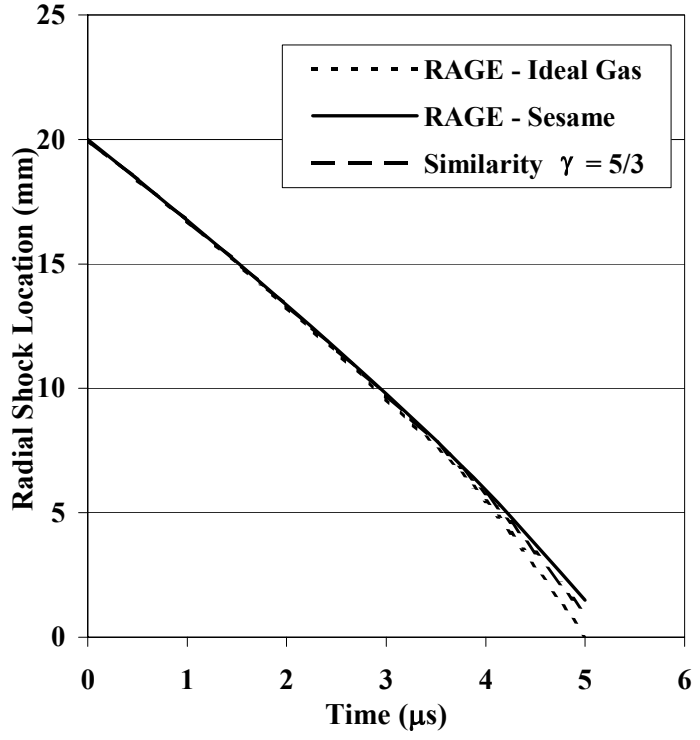

FIGURE 3. Results show and even larger level of discrepancy as the shock strength increases and the problem loses its ideal gas nature.

A closer examination of the importance of equation of state variations is possible with comparison to NTLX experimental results. The original mission of the NTLX series was to study shock convergence in simple convergent geometries. The second and fourth experiments of the series offset the Lucite core $4 \mathrm{~mm}$ from the liner driver center. This variation from the concentric alignment of Fig. 1 allows for shock timing measurements through a variable thickness Sn outer target. Comparisons of equation of state effects in a solid Sn target are now possible as seen in Fig. 4.

Figure 4 illustrates the difficulties in trying to draw a consensus from simulations when the same equation of state data produces different results as with RAGE and RAVEN. It is encouraging to see that a fit to NTLX data tracks well with the RAGE results with a slight $100 \mathrm{~ns}$ temporal offset. Timing discrepancies of this order are characteristic of all data from the experimental series. This error is associated with discrepancies in determining the absolute timing of the liner/target impact. The relative agreement of these results is very good, but more data is required before concise conclusions can be drawn. Figure 4 provides additional comparisons of RAGE simulations using a 
Grueneisen EOS with similarity solutions using $\gamma=$ 2 for Sn. The discrepancy between results indicates that we are in a moderately strong shock regime where SESAME shock data provides a better representation than the Grueneisen EOS.
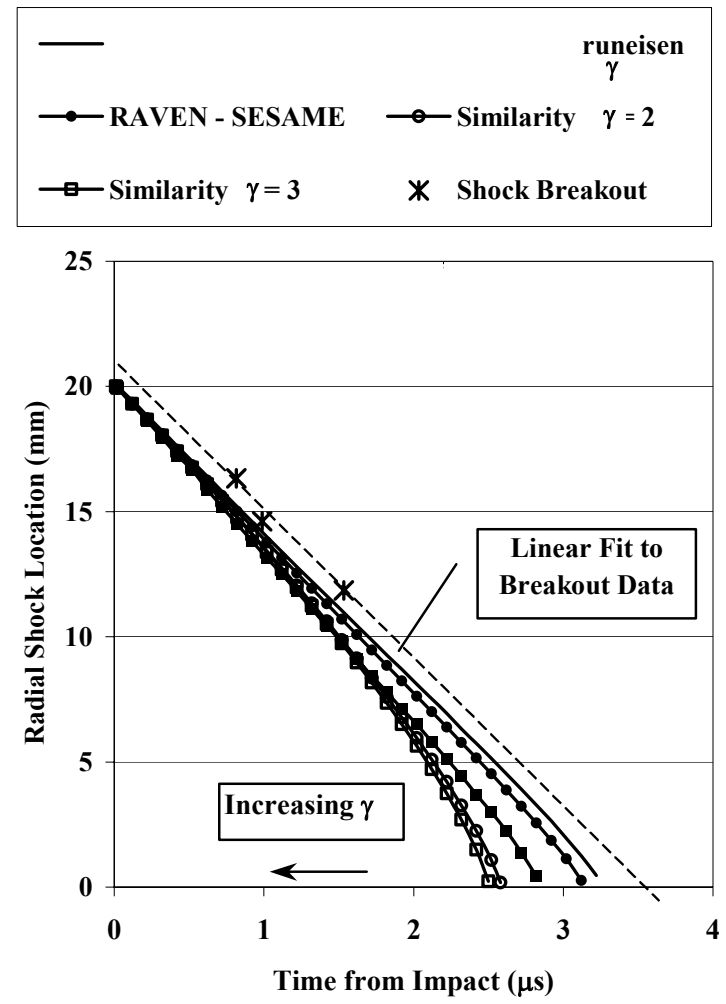

FIGURE 4. Comparisons of NTLX data with simulations show good relative agreement with RAGE data using the SESAME equation of state information.

\section{CONCLUSIONS}

With an increased interest in using numerical simulation as a predictive tool, it is necessary to ensure that there exists a sufficient level of confidence in general use codes. Computational tools must be capable of simulating relatively simple as well as more complex experiments. The current efforts compared simulations utilizing the RAVEN and RAGE codes with similarity solutions and data from the NTLX experimental series.
Under the limitations of an ideal gas at STP, a self-similar solution provides a good representation of shock motion in cylindrical geometry. However, the assumptions underlying the similarity solution break down as the shock strength increases. For strong shocks, it is necessary to rely on experimental data for code validation efforts.

The NTLX experiment was able to provide shock propagation data through a variable thickness Sn shell. This allows evaluation of both analytic (Grueneisen) and tabular (SESAME) equations of state in the RAGE hydro code. For the same initial conditions, RAVEN generates a slightly faster shock than the one produced by RAGE. Figure 4 showed that the NTLX data slightly favors the RAGE results. However, this is based on limited experimental results and additional data is required for better comparison.

Self-similar solutions for converging shocks exhibit very good agreement with calculations using RAVEN and RAGE when the gas can be described as a perfect gas. In this regime, similarity solutions provide a good mechanism of code validation except near the convergence axis. Near this axis, strengthening of the incident shock invalidates the perfect gas assumption and the similarity solution fails. Code validation efforts in convergent geometries will require further experimental data.

\section{REFERENCES}

1. Baltrusaitis, R.M., Gittings, M.L., Weaver, R.P., Benjamin, R.F. and Budzinski, J.M., Phys. Fluids 8, pp. 2471-2483 (1996).

2. Oliphant, T.A., RAVEN Physics Manual, Los Alamos National Laboratory, Los Alamos, NM, LA-8802-M (1981).

3. Oliphant, T.A. and Witte, K.H., RAVEN, Los Alamos National Laboratory, Los Alamos, NM, LA-10826 (1987).

4. Lyon, S.P. and Johnson, J.D., SESAME: the Los Alamos National Laboratory Equation of State Database, Los Alamos National Laboratory, Los Alamos, NM, LA-UR92-3407 (1992).

5. Steinberg, D.J., Cochran, S.G. and Guinan, M.W., J. Appl. Phys. 51, pp. 1498-1504 (1980).

6. Guderley, G., Luftfahrtforsch 19, pp.302-312 (1942).

7. Chisnell, R.F., J. Fluid Mech. 354, pp. 357-375 (1998).

8. Whithan, G.B., Linear and Nonlinear Waves, Wiley, New York, 1999, pp. 191-199. 